\title{
Constraints in Anaerobic Microbial Dechlorination, Fermentation, and Sulfate-Reduction Induced by High Concentrations of Tetrachloroethylene
}

\author{
Miho Yoshikawa $\mathbb{D} \cdot$ Ming Zhang $(\mathbb{D}$
}

Received: 29 March 2020 / Accepted: 7 July 2020 / Published online: 20 July 2020

(C) The Author(s) 2020

\begin{abstract}
Anaerobic bioremediation of tetrachloroethylene (PCE) under high concentration conditions is difficult. Anaerobic dechlorination of PCE occurs with synergetic reactions, fermentation, and sulfatereduction; however, the way in which high concentrations of PCE affects these reactions is still poorly understood. This study aims to elucidate how high concentrations of PCE affect fermentation and sulfate-reduction, as well as PCE dechlorination. Laboratory dechlorination tests were performed using a wide concentration range of PCE between 2 and $125 \mathrm{mg} / \mathrm{L}$ added to a microbial consortium that had been continuously cultivated in the laboratory and completely dechlorinated PCE for over 4 years. Fermentation of lactate, reduction of sulfate, and dechlorination of PCE were monitored in addition to microbial activities based on RNA. All three reactions, fermentation, sulfate-reduction, and PCE dechlorination were observed to be inhibited. The inhibition for fermentation, sulfate-reduction, and dechlorination occurred when PCE concentrations were higher than 125,75 , and $30 \mathrm{mg} / \mathrm{L}$, respectively. The fermenter, Anaerotignum, and the sulfate-reducer, Desulfosporosinus, were active when the dechlorination was inhibited with $30 \mathrm{mg} / \mathrm{L}$ of PCE. These
\end{abstract}

M. Yoshikawa $(\bowtie) \cdot$ M. Zhang

Geological Survey of Japan, National Institute of Advanced Industrial Science and Technology (AIST), 1-1-1, Higashi,

Tsukuba, Ibaraki 305-8567, Japan

e-mail: m.yoshikawa@aist.go.jp findings suggest that there is interference of PCE dechlorination, despite the occurrence of fermentation and sulfate reduction. Bioaugmentation with a PCE dechlorinator that is tolerant to high PCE concentrations can be a possible solution for bioremediation of PCE when its concentrations are greater than $30 \mathrm{mg} / \mathrm{L}$.

Keywords Biodegradation - High concentration · Chloroethylenes $\cdot$ Microbial community $\cdot$ RNA

\section{Introduction}

Soil and groundwater in many industrial sites are contaminated with high concentrations of tetrachloroethylene (PCE) (US EPA 2003). PCE has been used as an effective solvent in the dry cleaning and manufacturing industries (US EPA 2002; Zhang and Yoshikawa 2016). Once PCE is released into subsurface due to illegal and/or careless disposal from these industries, the high concentration of contaminant slowly partitions into groundwater; then, it can migrate long distances dependent on geological characteristics of the site and pollute large area (EPA 1998; US EPA 2003). Thus, besides the removal of pollutant source, remediation of the area near the pollutant source with high concentrations is a significant issue. Bioremediation, the remediation of soil or water using microorganisms, is considered difficult in instances where there is a high concentration of PCE (US EPA 2003; Juwarkar et al. 2010; 
Zhang and Yoshikawa 2016). In such cases, remediation conventionally consists of other engineering technologies such as in situ hydraulic containment, off-site disposal, and institution controls. Bioremediation has been largely selected for use in groundwater with a low concentration of pollutants (US EPA 2017). While the difficulty of bioremediation of PCE at high concentrations is known in practice, the reason for this difficulty at the microbial community scale has not been fully understood.

Anaerobic dechlorination of PCE occurs due to microbial degradation alongside other microbial activities. One essential role played by other microorganisms is fermentation (Parsons 2004). To enable the reductive dechlorination of chloroethylenes, such as PCE and its toxic intermediate products; tetrachloroethylene (TCE), cis-dichloroethylene (cisDCE), and vinyl chloride (VC), fermenting substrates to organic acids and producing molecular hydrogen are indispensable. The organic acids are used as carbon sources and/or electron donors for the dechlorinators of chloroethylenes, and molecular hydrogen is also used as their electron donor (e.g., Scholz-Muramatsu et al. 1995; Krumholz 1997; Sung et al. 2006; Löffler et al. 2013). Microbes also play an essential role in lowering the oxidationreduction potential (ORP). Sulfate-reducing bacteria support the reductive dechlorination due to decreasing the ORP (Parsons 2004). With a declining ORP, the rate of PCE dechlorination increases (Hirl and Irvine 1997). A previous laboratory study demonstrated the inhibition of anaerobic dechlorination of PCE when its concentration is higher than $90 \mathrm{mg} / \mathrm{L}$ with PCE dechlorinating isolates (Amos et al. 2007). However, the mechanisms at play that impact the synergetic reactions and PCE dechlorination in the microbial community are still poorly understood.

In this study, we aimed to reveal the effects of high PCE concentrations on the microbial community. Laboratory degradation tests with a wide PCE concentration range between 2 and $125 \mathrm{mg} / \mathrm{L}$ were carried out with a microbial consortium that had been continuously cultivated in the laboratory and completely dechlorinated PCE for over 4 years. We analyzed the changes in microbial structure at the RNA level as well as the fermentation, sulfate reduction, and PCE dechlorination processes, to elucidate whether and how high concentrations of PCE influences these processes in the microbial consortium.

\section{Materials and Methods}

\subsection{Biodegradation Tests}

\subsubsection{Enrichment Culture as Microbial Source}

A soil sample was collected from a site contaminated with toxic volatile organic compounds including PCE and other contaminants (Yoshikawa et al. 2017). The soil was mixed with a mineral solution and anaerobically incubated with PCE at a concentration of $30 \mathrm{mg} / \mathrm{L}$ as per Yoshikawa et al. (2016, 2017). Sodium acetate, sodium lactate, yeast extract, and methanol were added as carbon sources and/or electron donors. Following dechlorination of chloroethylenes, a portion of culture was transferred to the fresh mineral solution at a ratio of 1:5 (v/v) to enrich the microorganisms degrading chloroethylenes. The enrichment culture was allowed to stably dechlorinate chloroethylenes and produce ethylene for over 4 years. VC is more toxic compared with its parent compound, and excessive accumulation of VC or the degradation ends by VC should be avoided. Previous studies (Yoshikawa et al. 2017) showed that when the concentration of PCE was less than $30 \mathrm{mg} / \mathrm{L}$ and with the existence of Dehalococcoides, PCE could be completely degraded to ethylene which is a harmless substance.

\subsubsection{Biodegradation Test with Different Concentrations of PCE}

An aliquot of $32 \mathrm{~mL}$ of the enrichment culture and $160 \mathrm{~mL}$ of the fresh mineral solution were mixed in each of $220 \mathrm{~mL}$ test bottles. Each bottle was sealed with an aluminum cap and a Teflon-coated butyl lubber septum, followed by replacement of the headspace gas with $\mathrm{N}_{2}$. Then, sodium lactate, sodium acetate, yeast extract, and methanol were added. PCE was added for concentrations of $2,6,30,75$, and $125 \mathrm{mg} / \mathrm{L}$, which equated to $2.4,7.1,35.5,88.7$, and $147.9 \mu \mathrm{mol} / \mathrm{bottle}$, respectively. We named the conditions PCE-2, PCE-6, PCE-30, PCE-75, and PCE-125 to correspond to the 2, $6,30,75$, and $125 \mathrm{mg} / \mathrm{L}$ PCE concentrations, respectively. The test bottles were placed in a dark shaking incubator $\left(150 \mathrm{rpm}, 30^{\circ} \mathrm{C}\right)$. We performed the biodegradation test in triplicate. We also prepared bottles with no PCE added to analyze initial concentrations of organic acids, $\mathrm{SO}_{4}{ }^{2-}$, and the microbial community. This 
dataset represented day 0 in this study. We also prepared bottles with $30 \mathrm{mg} / \mathrm{L}$ of PCE as sterile controls.

\subsection{Changes in Chemical Properties}

\subsubsection{Fermentation in Culture Solution}

To evaluate the fermentation of organic matter, organic acids including lactate, acetate, and propionate were analyzed during the experimental timeframe. We collected $1 \mathrm{~mL}$ of culture solution periodically from each of the test bottles to a plastic tube. This was then centrifuged (14,000 rpm, $\left.4{ }^{\circ} \mathrm{C}, 4 \mathrm{~min}\right)$ to divide the supernatant and pellet. The supernatant was filtered with a $0.22-\mu \mathrm{m}$ polytetrafluoroethylene (PTFE) syringe filter (Membrane Solutions, Plano, TX, USA) for the analysis of organic acids. A high-performance liquid chromatograph (HPLC) system was used (Shimadzu) with two equivalent columns installed (Shim-Pack SCR-102H, Shimadzu) to separate the organic acids accurately. The organic acids were detected with a COD-10A detector (Shimadzu) using $0.005 \mathrm{mmol} / \mathrm{L}$ detection limits. Pyruvate and succinate were also analyzed, but the concentrations of them were below the detection limits and not shown in this paper. The pellet left in the plastic tube was used for subsequent RNA extraction.

\subsubsection{Sulfate Reduction in Culture Solution}

We monitored $\mathrm{SO}_{4}{ }^{2-}$ concentrations over time to investigate sulfate reduction. The filtered supernatant, as described in Section 2.2.1, was applied to analyze $\mathrm{SO}_{4}{ }^{2-}$ with an ion chromatography (IC-2010, Tosoh, Tokyo, Japan) installed with a column (TSKgel SuperIC-Anion HS, Tosoh). The detection limit was $0.001 \mathrm{mmol} / \mathrm{L}$.

\subsubsection{Dechlorination of PCE}

We monitored PCE concentrations, its intermediate products, and the harmless non-chlorinated product, ethylene, in the headspace gas of the test bottle. We analyzed PCE and its intermediate products with a gas chromatography (GC-2014, Shimadzu, Kyoto, Japan) and a gas chromatography-mass spectrometry (GCMS-QP2010 Ultra, Shimadzu), and ethylene with a gas chromatography (GC-2014). The selection of GC or GC-MS was based on the concentrations of PCE and its intermediate products to be analyzed. GC and GCMS were used to analyze PCE and its intermediate products when their concentrations were greater than $1 \mathrm{mg} / \mathrm{L}$, and less than $1 \mathrm{mg} / \mathrm{L}$, respectively. Capillary columns of GS-Q (0.53 mm diameter, 30-m length; Agilent Technologies, Santa Clara, CA, USA) and RTX-624 (0.25 mm diameter, 60-m length, Restek, Bellefonte, PA, USA) were used for the separation in GC and GC-MS, respectively. For the GC analysis, the column temperature was maintained at $80^{\circ} \mathrm{C}$ for $1.0 \mathrm{~min}$, then increased to $220{ }^{\circ} \mathrm{C}$ at a speed of $35^{\circ} \mathrm{C}$ per min, and maintained at $220^{\circ} \mathrm{C}$ for $2.0 \mathrm{~min}$. A 25 $200 \mu \mathrm{L}$ aliquot of headspace gas was withdrawn from each test bottle and injected to the GC manually. For the GC-MS analysis, the column temperature was maintained at $100{ }^{\circ} \mathrm{C}$ for $3.5 \mathrm{~min}$, increased to $170{ }^{\circ} \mathrm{C}$ at a speed of $20^{\circ} \mathrm{C}$ per min. A $25-100 \mu \mathrm{L}$ aliquot of headspace gas was withdrawn from each test bottle and injected to the GC-MS manually. We calculated concentrations in the culture solution using the Henry's law constant (Mackay and Shiu 1981). The Henry's law constants for the PCE, TCE, cis-DCE, VC, and ethylene at $25^{\circ} \mathrm{C}$ were $0.93,0.48,0.31,0.95$, and 8.76 , respectively. Because the constants are sensitive to the temperature, the room temperature was continuously controlled at $25^{\circ} \mathrm{C}$ during the analyses. The detection limit for the chloroethylenes with GC-MS were $0.001 \mathrm{mg} / \mathrm{L}$. This was equivalent to $0.001,0.001,0.002$, and $0.003 \mu \mathrm{mol} /$ bottle for PCE, TCE, cis-DCE, and VC, respectively, whereas that of ethylene analyzed with GC was $0.028 \mathrm{mg} / \mathrm{L}$, equivalent to $0.20 \mu \mathrm{mol} / \mathrm{bottle}$. To ensure the precision of gas analyses, the linearities of calibration curves were confirmed to be $R^{2}>0.97$ and $R^{2}>0.99$ for the GC and GC-MS analyses, respectively.

\subsection{Changes in Microbiological Properties}

\subsubsection{Extraction of RNA and Synthesis of cDNA}

We periodically extracted RNA to assess the microbial communities as active microbial assemblies. The pellet collected with the centrifugation described in Section 2.2.1 was soaked in RNAlater ${ }^{\mathrm{TM}}$ Stabilization Solution (Thermo Fisher Scientific, Waltham, MA, USA) and stored at $-80{ }^{\circ} \mathrm{C}$ until RNA extraction. Extraction was carried out using NucleoSpin ${ }^{\circledR}$ RNA (Macherey-Nagel, Düren, Germany), followed by additional DNA digestion with DNA-free ${ }^{\mathrm{TM}}$ Kit (Thermo Fisher Scientific). cDNA was synthesized from the RNA extract with PrimeScript ${ }^{\mathrm{TM}} 1$ st strand cDNA 
Synthesis Kit (Takara Bio, Kusatsu, Japan) containing random 6-mer primers.

\subsubsection{Next-Generation Sequencing-Based Amplicon Sequencing}

Microbial communities were determined with nextgeneration sequencing (NGS)-based amplicon sequencing of V4 region of $16 \mathrm{~S}$ rRNA transcripts. The region of the cDNA was amplified with universal primers containing an adapter sequence for Illumina sequencer, 515F and 806R (Caporaso et al. 2012) and PrimeSTAR ${ }^{\circledR}$ GXL DNA Polymerase (Takara Bio, Kusatsu, Japan). Following the purification of the first PCR products with magnetic beads, specifically AMPure XP beads (Beckman Coulter, Brea, CA, USA), products were applied to a second PCR to add index sequences. Purified second PCR products were sequenced 300-bp paired end with Illumina Miseq (Illumina, San Diego, CA, USA). We performed data processing of the sequence data using QIIME 1 (Caporaso et al. 2010) and mothur (v.1.42.3) (Schloss et al. 2009). Non-chimeric sequences obtained from the QIIME 1 procession were grouped into operational taxonomic units (OTUs, 97\% similarity) with the mothur program. As reference taxonomy to affiliate taxonomy of the sequences, EzBioCloud 16S database (Yoon et al. 2017) was applied to the sequences.

\section{Results}

\subsection{Fermentation of Organic Compounds}

The $10.2 \mathrm{mmol} / \mathrm{L}$ of lactate in the culture solutions in the PCE-2, PCE-6, PCE-30, and PCE-75 tests immediately reduced, and the concentration was under the detection limit within 3 days (Fig. 1a). PCE-125 had a slower reduction, taking 6 days to achieve a concentration under the detection limit (Fig. 1a). Alongside lactate reduction, increments in acetate and propionate concentrations occurred (Fig. $1 \mathrm{~b}$ and c). We observed an increment of acetate from 10.4 to $13.7,13.9,13.7$, and $13.4 \mathrm{mmol} / \mathrm{L}$ in PCE-2, PCE-6, PCE-30, and PCE-75, respectively, during days $0-3$ (Fig. 1b). The concentration of acetate in PCE-125 at day 3 was $12.0 \mathrm{mmol} / \mathrm{L}$, lower than that under other test conditions, and then increased to $13.9 \mathrm{mmol} / \mathrm{L}$ at day 6 . The increment of propionate had a similar tendency to that of acetate (Fig. 1c). The concentrations of propionate in PCE-2, PCE-6, PCE-30, and PCE-75 had increments from 2.4 to 8.2 , 8.2, 8.4, and $8.2 \mathrm{mmol} / \mathrm{L}$, respectively, during days $0-3$. Concentrations of propionate in PCE-125 were only $4.7 \mathrm{mmol} / \mathrm{L}$ at day 3 , which then increased to $8.3 \mathrm{mmol} / \mathrm{L}$ during days $3-6$.

\subsection{Sulfate Reduction}

The concentrations of $\mathrm{SO}_{4}{ }^{2-}$ in culture solutions reduced from 0.8 to $0.03 \mathrm{mmol} / \mathrm{L}$ during days 0-6 in PCE-2, PCE6, and PCE-30 tests (Fig. 2). In PCE-75, $\mathrm{SO}_{4}{ }^{2-}$ reduction was observed later, during days 3-9. The culture solution of PCE-125 kept the concentration of $\mathrm{SO}_{4}{ }^{2-}$ around $0.8 \mathrm{mmol} / \mathrm{L}$ until day 24 , then the test showed the reduction of $\mathrm{SO}_{4}{ }^{2-}$ within the following 10 days.

\subsection{Anaerobic Microbial Dechlorination of PCE}

PCE dechlorination occurred in the tests of PCE-2, PCE-6, and PCE-30 (Fig. 3a-c). PCE was sequentially dechlorinated; TCE, cis-DCE, and VC were produced. Ethylene production was then confirmed in the PCE-2 and PCE- 6 tests over the 34 days of the experiment. PCE dechlorination to TCE was observed within the initial 6 days in both PCE-2 and PCE- 6 tests, while slower PCE dechlorination continued in PCE-30 test until day 24. The dechlorination rates of PCE were $0.33,0.60$, and $0.19 \mu \mathrm{mol} / \mathrm{bottle} /$ day in PCE-2, PCE-6, and PCE-30 tests, respectively. When tests were performed with 75 and $125 \mathrm{mg} / \mathrm{L}$ of PCE in PCE-75 and PCE-125, PCE dechlorination did not occur within the experimental time frame (Fig. $3 \mathrm{~d}$ and e). The dechlorination of PCE did not occur in the sterile control bottles too (data not shown). The mass balance between VC and ethylene was not obvious (Fig. 3). One possible decomposition product, specifically ethane, of VC, was also analyzed but not detected during our experiments. This means that there would be unknown decomposition pathways and byproducts that were involved in the degradation reactions, and future research should further develop and confirm this phenomenon.

\subsection{Microbial Diversity Based on Transcripts of $16 \mathrm{~S}$ rRNA}

Microbial communities based on cDNA at genus level with 29,853 sequences in each community are shown in Fig. 4. Anaerotignum accounted for a large percentage 

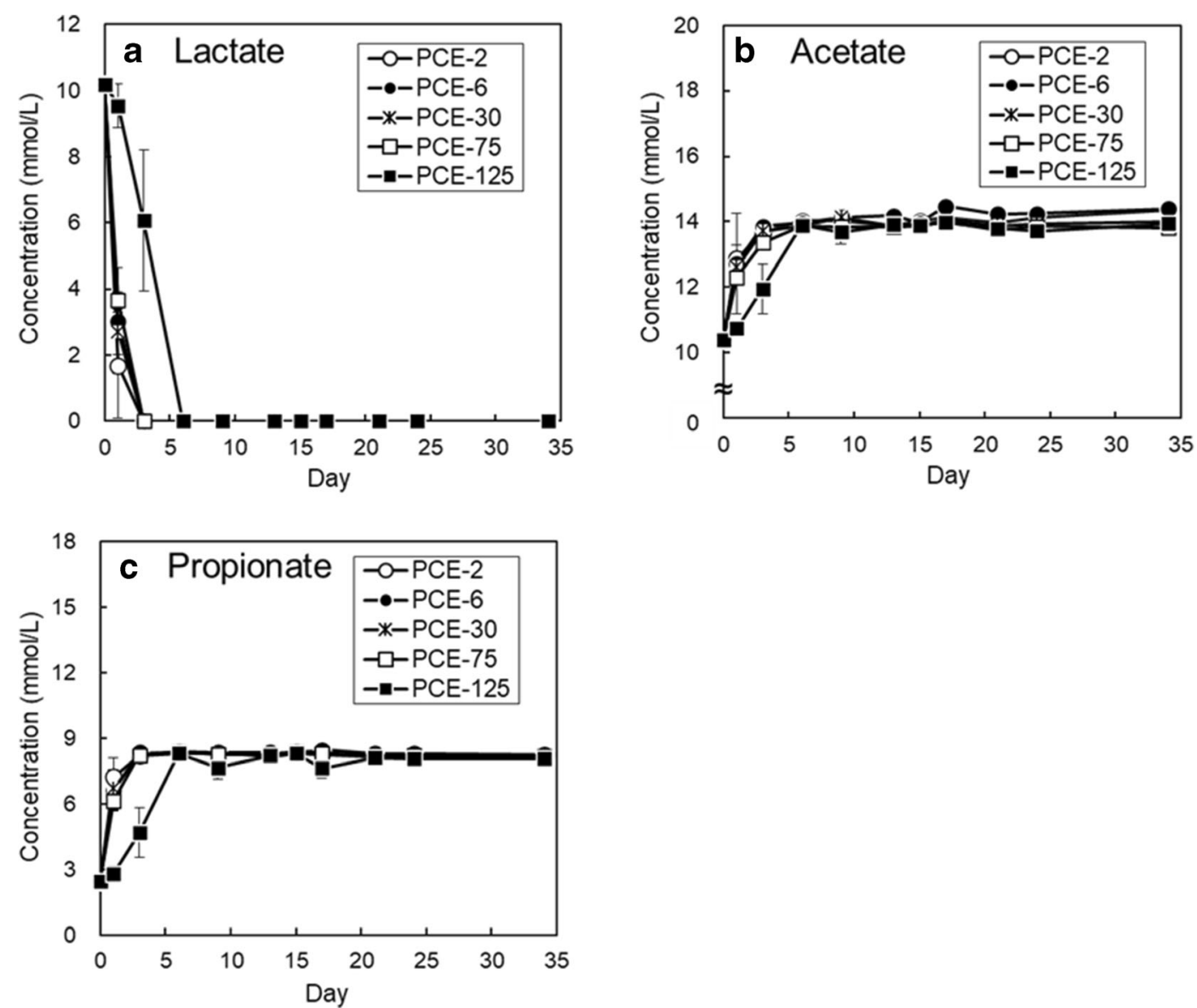

Fig. 1 Changes in concentrations of organic acids over the test period, a lactate, $\mathbf{b}$ acetate, and $\mathbf{c}$ propionate, with different initial concentrations of PCE. The error bars show standard errors

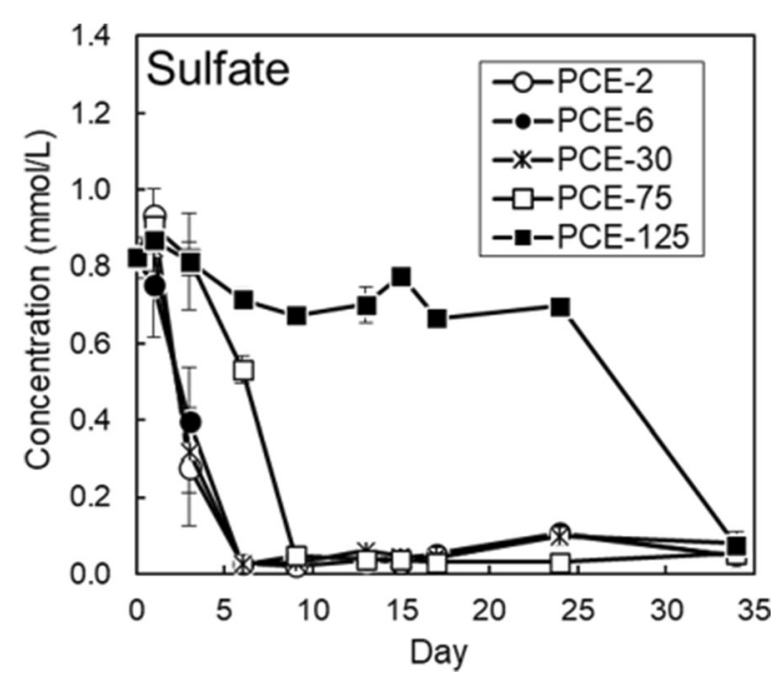

Fig. 2 Sulfate reduction under the conditions with different initial concentrations of PCE. The error bars show standard errors of the microbial communities in the PCE-2, PCE-6, PCE-30, and PCE-75 culture solutions during days 13. The genus was also predominant in microbial communities in PCE-125 culture solution during days 3-9. The proportion of Desulfosporosinus increased up to 19.0, 16.4, and 28.0\%, in PCE-2, PCE-6, and PCE-30 tests on day 3, respectively. As for PCE-75 and PCE125 tests, the genus increased to $27.4 \%$ in PCE-75 culture solution on day 6 , and $24.5 \%$ in PCE-125 culture solution on day 24. The genera of Sulfurospirillum, Desulfitomonas, and Geobacter were not observed in any of the communities. The genus Desulfitobacterium was observed in relatively low ratios from $0.01-0.04$, $0.01-0.03,0.02-0.05,0.03-0.12$, and $0.07-0.34 \%$, in PCE-2, PCE-6, PCE-30, PCE-75, and PCE-125 culture solutions during days 1-24, respectively. More specifically, the ratios of genus Desulfitobacterium were 0.02, $0.02,0.04,0.07$, and $0.08 \%$ in day 9 , and $0.02,0.01$, 

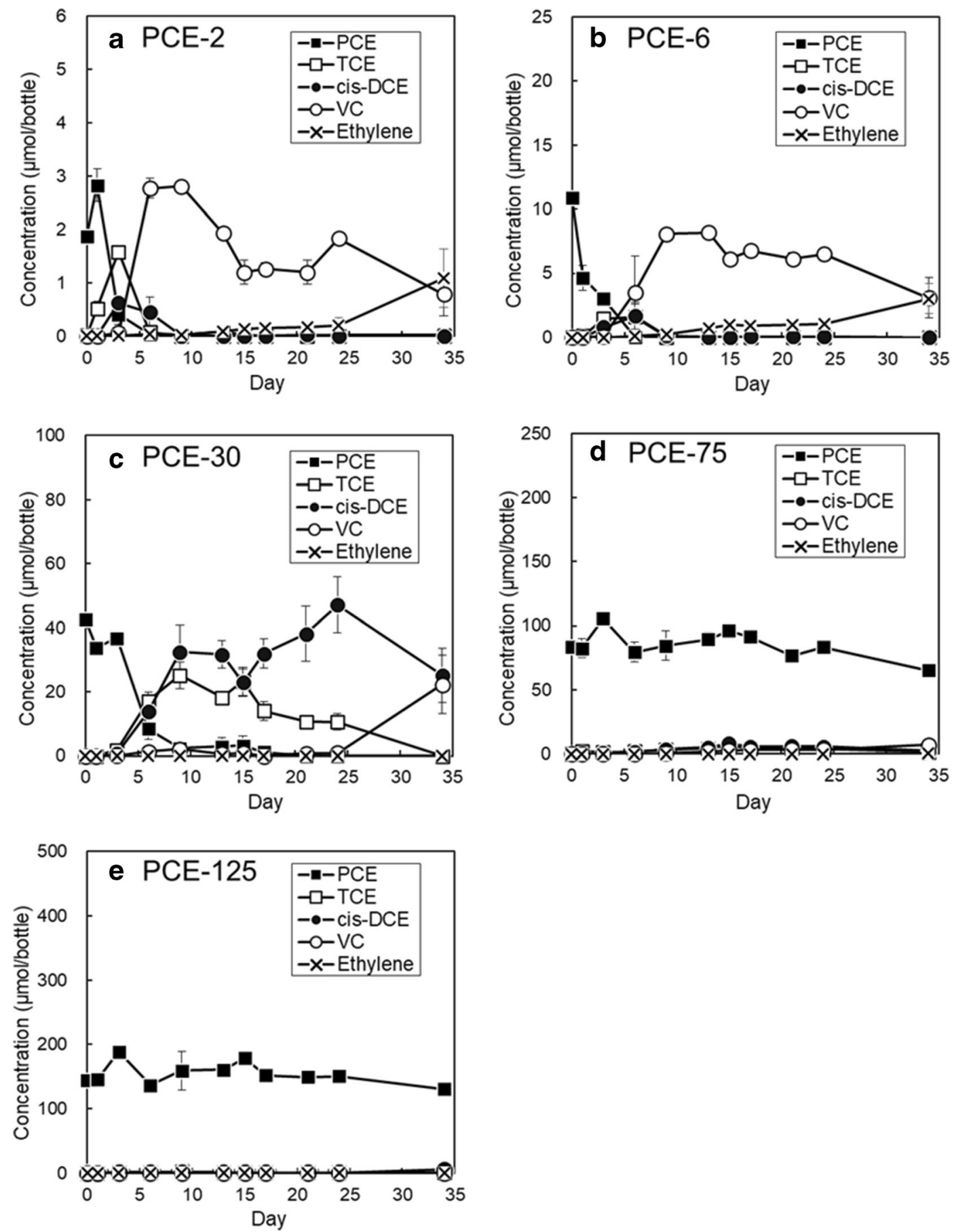

Fig. 3 Anaerobic dechlorination of PCE under different initial concentration conditions. The initial concentrations were a $2 \mathrm{mg} / \mathrm{L}$ (2.4 $\mu \mathrm{mol} /$ bottle), b $6 \mathrm{mg} / \mathrm{L}(7.1 \mu \mathrm{mol} /$ bottle $)$, c $30 \mathrm{mg} / \mathrm{L}$

(35.5 $\mu \mathrm{mol} /$ bottle), d $75 \mathrm{mg} / \mathrm{L}(88.7 \mu \mathrm{mol} /$ bottle $)$, and e $125 \mathrm{mg} /$ $\mathrm{L}(147.9 \mu \mathrm{mol} /$ bottle), respectively. The error bars show standard errors

$0.03,0.10$, and $0.16 \%$ in day 24, in PCE-2, PCE-6, PCE-30, PCE-75, and PCE-125 culture solutions, respectively. The abundance of genus Desulfitobacterium

in the PCE-125 culture solution was higher than that in any other culture solution with a lower PCE concentration. 

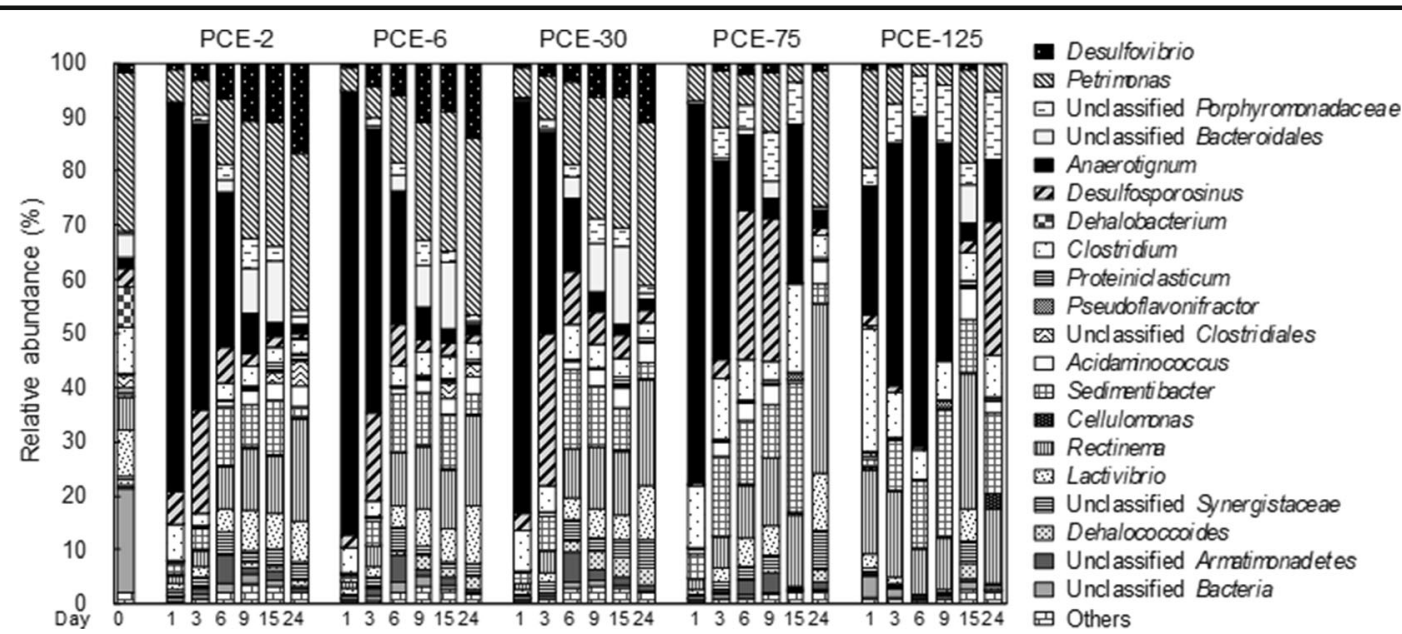

Fig. 4 The relative abundance of different microbial genus overtime based on cDNA amplicon sequencing. Initial PCE concentrations of PCE-2, $2 \mathrm{mg} / \mathrm{L}$ (2.4 $\mu \mathrm{mol} /$ bottle $) ;$ PCE- $6,6 \mathrm{mg} / \mathrm{L}$ (7.1 $\mu \mathrm{mol} / \mathrm{bottle})$; PCE-30, $30 \mathrm{mg} / \mathrm{L}(35.5 \mu \mathrm{mol} / \mathrm{bottle})$; PCE-75,

$75 \mathrm{mg} / \mathrm{L}(88.7 \mu \mathrm{mol} /$ bottle); and PCE-125, $125 \mathrm{mg} / \mathrm{L}$ $(147.9 \mu \mathrm{mol} / \mathrm{bottle})$. The less abundant genus $(<1 \%)$ were integrated into others

The structures of Anaerotignum, Desulfosporosinus, and Desulfitobacterium are shown in Fig. 5. The genus Anaerotignun included two major OTUs; OTU0001 and OTU0008 (Fig. 5a). The OTU0001 representative over $69.6 \%$ of relative abundance of Anaerotignun in the cDNA communities of PCE-2, PCE-6, PCE-30, and PCE-75, while the microbial community of PCE- 125 on day 6 contained $45.2 \%$ of OTU0001 and $54.3 \%$ of OTU0008. For Desulfosporosinus and Desulfitobacterium, OTU0004 and OTU0036, respectively, accounted for more than $90.5 \%$ of the relative abundance of the genus (Fig. $5 \mathrm{~b}$ and $\mathrm{c}$ ).

\section{Discussion}

\subsection{Effects of High PCE Concentrations on Lactate Fermentation}

Lactate reduction and the increment of cDNA abundance of Anaerotignum were induced simultaneously (Figs. 1 and 4). The representative sequence of Anaerotignum OTU0001 (Fig. 5) in this study was similar to Anaerotignum propionicum JCM1430 (Collins et al. 1994; Ueki et al. 2017) and Anaerotignum neopropionicum DSM3847 (Ueki et al. 2017) with $100.0 \%$ identicality. The strains JCM1430 and DSM3847 are gram-positive and spore-forming bacteria, which ferment lactate to acetate and propionate (Tholozan et al. 1992; Ueki et al. 2017). It is reasonable

that Anaerotignum was one of the fermenters in this study. Molecular hydrogen is produced during the fermentation process of organic acids including lactate, assisting with the anaerobic dechlorination of chlorinated ethylenes (Parsons 2004). In terms of hydrogen production, adequate conditions for production may have occurred when there was a PCE concentration of $75 \mathrm{~m} / \mathrm{L}$ or lower. Lactate fermentation and increments in the relative abundance of Anaerotignum was delayed for 3 days under a PCE concentration of $125 \mathrm{mg} / \mathrm{L} \mathrm{com-}$ pared to other concentrations (Figs. 1 and 4). Furthermore, with a PCE concentration of $125 \mathrm{mg} / \mathrm{L}$, Anaerotignum OTU0008 accounted for a higher percentage on day 6 , compared to other concentrations (Fig. 5). One possible reason for the continued fermentation under a PCE concentration of $125 \mathrm{mg} / \mathrm{L}$ may be due to fermentation by a more PCE-tolerant strain, Anaerotignum OTU0008, compared to Anaerotignum OTU0001.

\subsection{Impact of High PCE Concentrations on Sulfate Reduction}

The decreasing $\mathrm{SO}_{4}{ }^{2-}$ and increasing proportion of the genus Desulfosporosinus occurred concurrently when PCE concentrations were between 2 and $75 \mathrm{mg} / \mathrm{L}$ (Figs. 2 and 4). The obtained sequence of Desulfosporosinus OTU0004 was close to Desulfosporosinus meridiei S5 (Robertson et al. 2001) with $99.6 \%$ of identicality. The strain is gram-negative/ 
a Anaerotignum

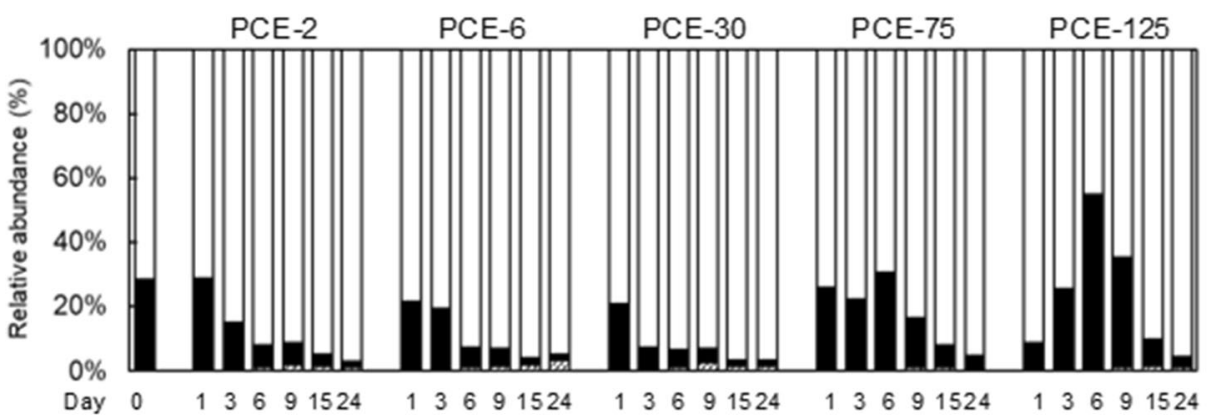

口OTU0001

- OTU0008

口OTU0047

øOthers

b Desulfosporosinus

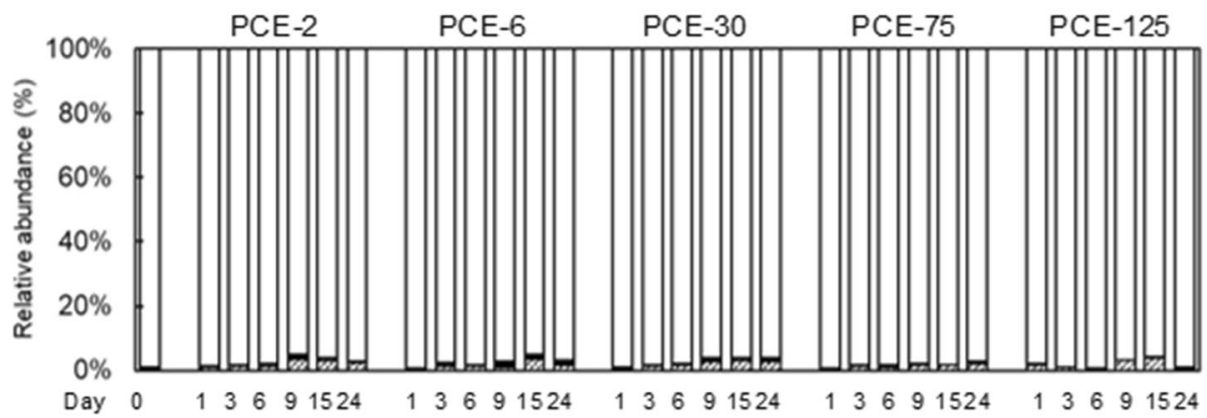

口OTU0004

-OTU0056

口OTU0061

øOthers

C Desulfitobacterium

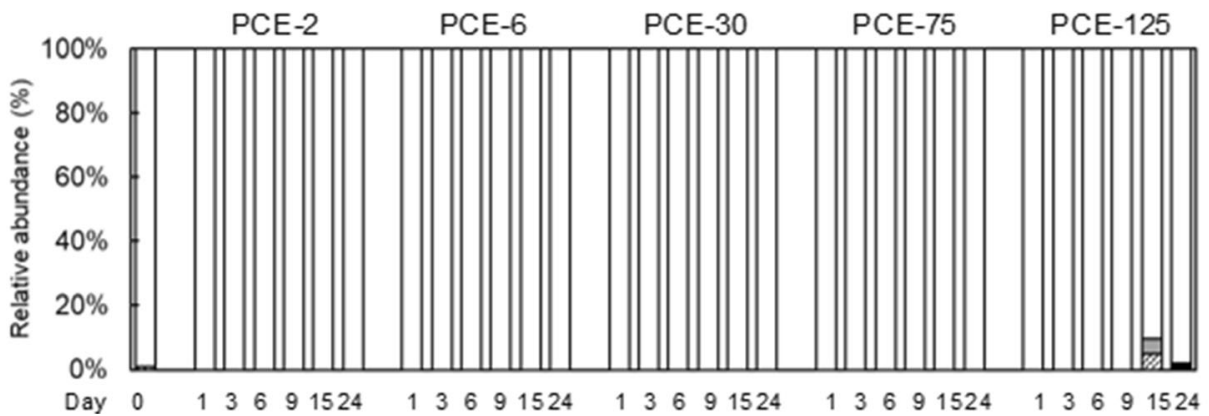

口OTU0036

-OTU1423

口OTU2885

冈Others

Fig. 5 The relative abundances of the top three OTUs overtime classified into a Anaerotignum, b Desulfosporosinus, and c Desulfitobacterium. PCE-2, $2 \mathrm{mg} / \mathrm{L}(2.4 \mu \mathrm{mol} / \mathrm{bottle})$ of initial concentration of PCE; PCE- $6,6 \mathrm{mg} / \mathrm{L}(7.1 \mu \mathrm{mol} / \mathrm{bottle})$ of initial concentration of PCE; PCE-30, $30 \mathrm{mg} / \mathrm{L}(35.5 \mu \mathrm{mol} / \mathrm{bottle})$ of initial concentration of PCE; PCE-75, $75 \mathrm{mg} / \mathrm{L}(88.7 \mu \mathrm{mol} / \mathrm{bottle})$ of initial concentration of PCE; and PCE-125, PCE-125 with $125 \mathrm{mg} / \mathrm{L}(147.9 \mu \mathrm{mol} /$ bottle) of initial concentration of PCE. The less abundant genus $(<1 \%)$ were integrated into others

As opposed to Anaerotignum, the fermenter, the same Desulfosporosinus, OTU0004 was activated despite the PCE concentration (Fig. 5b). The results indicate that the same species of Desulfosporosinus may adapt to high PCE concentrations after a lag time. As reported with gramnegative bacteria, there may be some mechanisms that can be considered for adaptation. For example, modifying the cell membrane to decrease the permeability of the solvent, activating solvent efflux pumps to reduce accumulation of 
the solvent in a cell, and inactivating the solvent with an enzymatic reaction (Sardessai and Bhosle 2002; Torres et al. 2011).

\subsection{Constraints in Anaerobic Microbial Dechlorination of PCE at High PCE Concentrations}

Anaerobic PCE dechlorination was inhibited when PCE concentrations were above $30 \mathrm{mg} / \mathrm{L}$ and completely interfered when PCE concentrations were above $75 \mathrm{mg} / \mathrm{L}$ (Fig. 3). The solubility of PCE at $20^{\circ} \mathrm{C}$ is about $150 \mathrm{mg} / \mathrm{L}$. This means that bioremediation is basically not applicable to remediate the source zone and its surrounding areas where concentrations of PCE are higher than $30 \mathrm{mg} / \mathrm{L}$. However, the bioremediation can be effectively used to remediate the groundwater contaminated plume where the concentrations of PCE are less than $30 \mathrm{mg} / \mathrm{L}$. Other technologies should be adopted to clean up the source zone and its surrounding areas, but discussion on this topic is beyond the scope of this paper.

This study was carried out with a microbial consortium, though the results were similar to those tested with PCE dechlorinating isolates (Amos et al. 2007). Amos et al. (2007) found that the dechlorination of PCE for PCE concentrations above $90 \mathrm{mg} / \mathrm{L}$ had ceased when they tested with Sulfurospirillum multivorans, Desulfuromonas michiganensis BB1, Geobacter lovleyi SZ, and Desulfitobacterium sp. Viet1. The representative sequence of Desulfitobacterium OTU0036 (Fig. 5c) was similar to known dechlorinating strains, Desulfitobacterium sp. Viet1 (100.0\%, Löffler et al. 1997), Desulfitobacterium hafniense PCE-S (99.2\%, Miller et al. 1997), and Desulfitobacterium hafniense Y51 (99.2\%, Suyama et al. 2001). The cDNA-based communities in this study did not contain similar species to known PCE degrading strains of S. multivorans (Neumann et al. 1994; Scholz-Muramatsu et al. 1995), Desulfuromonas chloroethenica (Krumholz 1997), D. michiganensis (Sung et al. 2003), and G. lovleyi (Sung et al. 2006). Considering previous studies, one possible dechlorinating bacterium in this study could be Desulfitobacterium. The genus of Desulfitobacterium can also utilize the sulfate-reducing product, sulfite, as an electron acceptor (Christiansen and Ahring 1996). Our results suggest that anaerobic dechlorination is constrained with high PCE concentrations even when dechlorinating microorganisms were alive with other electron acceptors. Future studies need to be performed to investigate the expression of the functional genes encoding PCE dehalogenase and sulfite reductase. The outcomes of such studies will facilitate better understanding on the mechanisms of inhibition of dechlorination associated with high PCE concentrations.

\section{Conclusions}

We found that the inhibition of PCE dechlorination with high PCE concentrations occurs even when synergetic anaerobic reactions of fermentation and sulfate-reduction proceed. Among fermenters and sulfate reducers, strategies to overcome the toxicity of PCE were observed; different species of fermenters, Anaerotignum, were activated, while the same species of sulfate-reducer, Desulfosporosinus, adapted to the high concentrations after a lag time. However, we did not observe such strategies on dechlorinating bacteria during the experiment. To perform successful bioremediation of PCE under concentrations over $30 \mathrm{mg} /$ $\mathrm{L}$, the bioaugmentation of dechlorinating microorganisms tolerant to high concentrations of PCE is considered as one possible solution.

Funding Information This study was partially supported by the Environment Research and Technology Development Fund (JPMEERF20175001) of the Environmental Restoration and Conservation Agency of Japan.Data AvailabilityThe data that support the findings of this study are available from the corresponding author, upon reasonable request.

\section{Compliance with Ethical Standards}

Conflict of Interest The authors declare that there is no conflict of interest.

Code Availability Not applicable.

Open Access This article is licensed under a Creative Commons Attribution 4.0 International License, which permits use, sharing, adaptation, distribution and reproduction in any medium or format, as long as you give appropriate credit to the original author(s) and the source, provide a link to the Creative Commons licence, and indicate if changes were made. The images or other third party material in this article are included in the article's Creative Commons licence, unless indicated otherwise in a credit line to the material. If material is not included in the article's Creative Commons licence and your intended use is not permitted by statutory regulation or exceeds the permitted use, you will need to obtain permission directly from the copyright holder. To view a copy of this licence, visit http://creativecommons.org/licenses/by/4.0/. 


\section{References}

Amos, B. K., Christ, J. A., Abriola, L. M., Pennell, K. D., \& Löffler, F. E. (2007). Experimental evaluation and mathematical modeling of microbially enhanced tetrachloroethene (PCE) dissolution. Environmental Science and Technology, 41(3), 963-970.

Caporaso, J. G., Kuczynski, J., Stombaugh, J., Bittinger, K., Bushman, F. D., Costello, E. K., et al. (2010). QIIME allows analysis of high-throughput community sequencing data. Nature Publishing Group, 7(5), 335-336.

Caporaso, J. G., Lauber, C. L., Walters, W. A., Berg-Lyons, D., Huntley, J., Fierer, N., et al. (2012). Ultra-high-throughput microbial community analysis on the Illumina HiSeq and MiSeq platforms. The ISME Journal, 6(8), 1621-1624.

Christiansen, N., \& Ahring, B. K. (1996). Desulfitobacterium hafniense sp. nov., an anaerobic, reductively dechlorinating bacterium. International Journal of Systematic Bacteriology, $46(2), 442-448$.

Collins, M. D., Lawson, P. A., Willems, A., Cordoba, J. J., Fernandez-Garayzabal, J., Garcia, P., et al. (1994). The phylogeny of the genus Clostridium: proposal of five new genera and eleven new species combinations. International Journal of Systematic Bacteriology, 44(4), 812-826.

Hirl, P. J., \& Irvine, R. L. (1997). Anaerobic reductive dechlorination of perchloroethylene (PCE). In R. L. Irvine \& S. K. Sikdar (Eds.), Biodegradation technology developments: principles and practice, Volume II (pp. 329-351). Boca Raton: CRC Press.

Juwarkar, A. A., Singh, S. K., \& Mudhoo, A. (2010). A comprehensive overview of elements in bioremediation. Reviews in Environmental Science and Biotechnology, 9, 215-288.

Krumholz, L. R. (1997). Desulfuromonas chloroethenica sp. nov. uses tetrachloroethylene and trichloroethylene as electron acceptors. International Journal of Systematic Bacteriology, 47(4), 1262-1263.

Löffler, F. E., Ritalahti, K. M., \& Tiedje, J. M. (1997). Dechlorination of chloroethenes is inhibited by 2-bromoethanesulfonate in the absence of methanogens. Applied and Environmental Microbiology, 63(12), 4982-4985.

Löffler, F. E., Yan, J., Ritalahti, K. M., Adrian, L., Edwards, E. A., Konstantinidis, K. T., et al. (2013). Dehalococcoides mccartyi gen. nov., sp. nov., obligately organohaliderespiring anaerobic bacteria relevant to halogen cycling and bioremediation, belong to a novel bacterial class, Dehalococcoidia classis nov., order Dehalococcoidales ord. nov. and family Dehalococcoidaceae fam. nov., within the phylum Chloroflexi. International Journal of Systematic and Evolutionary Microbiology, 63(2), 625-635.

Mackay, D., \& Shiu, W. Y. (1981) A critical review of Henry's law constants for chemicals of environmental interest. Journal of Physical and Chemical Reference Data, 10 (4): 1175-1199

Miller, E., Wohlfarth, G., \& Diekert, G. (1997). Comparative studies on tetrachloroethene reductive dechlorination mediated by Desulfitobacterium sp. strain PCE-S. Archives of Microbiology, 168, 513-519.

Neumann, A., Scholz-Muramatsu, H., \& Diekert, G. (1994). Tetrachloroethene metabolism of Dehalospirillum multivorans. Archives of Microbiology., 162, 295-301.
Parsons. (2004). Principles and practices of enhanced anaerobic bioremediation of chlorinated solvents. AFCEE, NFEC, ESTCP..

Robertson, W. J., Bowman, J. P., Franzmann, P. D., \& Mee, B. J. (2001). Desulfosporosinus meridiei sp. nov., a spore-forming sulfate-reducing bacterium isolated from gasolenecontaminated groundwater. International Journal of Systematic and Evolutionary Microbiology, 51(1), 133-140.

Sardessai, Y., \& Bhosle, S. (2002). Tolerance of bacteria to organic solvents. Research in Microbiology, 153(5), 263268.

Schloss, P. D., Westcott, S. L., Ryabin, T., Hall, J. R., Hartmann, M., Hollister, E. B., et al. (2009). Introducing mothur: Open-source, platform-independent, community-supported software for describing and comparing microbial communities. Applied and Environmental Microbiology, 75(23), 7537-7541.

Scholz-Muramatsu, H., Neumann, A., Meßmer, M., Moore, E., \& Diekert, G. (1995). Isolation and characterization of Dehalospirillum multivorans gen. nov., sp. nov., a tetrachloroethene-utilizing, strictly anaerobic bacterium. Archives of Microbiology, 163, 48-56.

Sung, Y., Ritalahti, K. M., Sanford, R. A., Urbance, J. W., Flynn, S. J., Tiedje, J. M., et al. (2003). Characterization of two tetrachloroethene-reducing, acetate-oxidizing anaerobic bacteria and their description as Desulfuromonas michiganensis sp. nov. Applied and Environmental Microbiology, 69(5), 2964-2974.

Sung, Y., Fletcher, K. E., Ritalahti, K. M., Apkarian, R. P., RamosHernández, N., Sanford, R. A., et al. (2006). Geobacter lovleyi sp. nov. strain SZ, a novel metal-reducing and tetrachloroethenedechlorinating bacterium. Applied and Environmental Microbiology, 72(4), 2775-2782.

Suyama, A., Iwakiri, R., Kai, K., Tokunaga, T., Sera, N., \& Furukawa, K. (2001). Isolation and characterization of Desulfitobacterium sp. strain Y51 capable of efficient dehalogenation of tetrachloroethene and polychloroethanes. Bioscience, Biotechnology, and Biochemistry, 65(7), 1474 1481.

Tholozan, J. L., Touzel, J. P., Samain, E., Grivet, J. P., Prensier, G., \& Albagnac, G. (1992). Clostridium neopropionicum sp. nov., a strict anaerobic bacterium fermenting ethanol to propionate through acrylate pathway. Archives of Microbiology, 157, 249-257.

Torres, S., Pandey, A., \& Castro, G. R. (2011). Organic solvent adaptation of Gram positive bacteria: applications and biotechnological potentials. Biotechnology Advances, 29(4), 442-452.

Ueki, A., Goto, K., Ohtaki, Y., Kaku, N., \& Ueki, K. (2017). Description of Anaerotignum aminivorans gen. nov., sp. nov., a strictly anaerobic, amino-acid-decomposing bacterium isolated from a methanogenic reactor, and reclassification of Clostridium propionicum, Clostridium neopropionicum and Clostridium lactatifermentans as species of the genus Anaerotignum. International Journal of Systematic and Evolutionary Microbiology, 67(10), 4146-4153.

United States Environmental Protection Agency (US EPA). (1998). Technical protocol for evaluating natural attenuation of chlorinated solvents in ground water. Washington, DC: United States Environmental Protection Agency EPA/ 600/R-98/128. 
United States Environmental Protection Agency (US EPA). (2002). Engineered approaches to in situ bioremediation of chlorinated solvents: fundamentals and field applications. Washington, DC: United States Environmental Protection Agency EPA 542-R-00-008.

United States Environmental Protection Agency (US EPA). (2003). The DNAPL remediation challenge: is there a case for source depletion? Washington, DC: United States Environmental Protection Agency EPA/600/R-03/143.

United States Environmental Protection Agency (US EPA). (2017). Superfund remedy report (15th ed.). Washington, DC: United States Environmental Protection Agency EPA 542-R-17-001.

Yoon, S. H., Ha, S. M., Kwon, S., Lim, J., Kim, Y., Seo, H., \& Chun, J. (2017). Introducing EzBioCloud: a taxonomically united database of 16S rRNA gene sequences and wholegenome assemblies. International Journal of Systematic and Evolutionary Microbiology, 67(5), 1613-1617.
Yoshikawa, M., Zhang, M., \& Toyota, K. (2016). Enhancement and biological characteristics related to aerobic biodegradation of toluene with co-existence of benzene. Water, Air, \& Soil Pollution, 227, 340.

Yoshikawa, M., Zhang, M., \& Toyota, K. (2017). Integrated anaerobic-aerobic biodegradation of multiple contaminants including chlorinated ethylenes, benzene, toluene, and dichloromethane. Water, Air, \& Soil Pollution, 228, 25.

Zhang, M., \& Yoshikawa, M. (2016). An overview of remediation technologies for sites contaminated with volatile organic compounds. InProceedings of GeoChicago 2016: Sustainability, Energy, and the Geoenvironment (pp. 295-301).

Publisher's Note Springer Nature remains neutral with regard to jurisdictional claims in published maps and institutional affiliations. 